\title{
Some factors influencing quality of spontaneous or induced sputum for inflammatory cell analysis
}

\author{
M.L. Bartoli, E. Bacci, S. Cianchetti, F.L. Dente, A. Di Franco, \\ B. Vagaggini, M. De Santis, E. Masino, P.L. Paggiaro
}

ABSTRACT: Some factors influencing quality of spontaneous or induced sputum for inflammatory cell analysis. M.L. Bartoli, E. Bacci, S. Cianchetti, F.L. Dente, A. Di Franco, B. Vagaggini, M. De Santis, E. Masino, P.L. Paggiaro.

Aim. To find some simple clinical factors which can predict the quality of the sputum samples obtained in a large group of asthmatic subjects.

Methods. We compared the presence of sputum productive cough in the days preceding the test, easiness in expectoration during the test, and sputum macroscopic aspect (presence of visible plugs) with the quality of slides obtained from sputum processing. We also monitored changes in the quality in patients who repeated sputum collection several times, comparing those whose first sample was adequate with those whose first sample was inadequate. We analysed 547 sputum samples obtained from 238 asthmatic patients. Sputum was processed using the whole sample method.

Results. Patients with productive cough in the days preceding the test and easy expectoration during the test produced a higher percentage of adequate samples than those without productive cough $(86 \%$ vs $76 \%, p=0.01)$ and with difficulty in expectoration $(85 \%$ vs $63 \%, p=0.0001)$. "Good" macroscopic samples were associated with better quality of slides $(91 \%$ vs $38 \%, p=0.0001)$. Patients with inadequate first sample $(n=40)$ had a higher percentage of inadequate samples $(55 \%)$ in the subsequent tests than patients $(\mathrm{n}=115)$ with adequate first sample $(8 \%)$.

Conclusions. Patients with increased airway secretions in the days preceding the test, easy expectoration and "good" macroscopic aspect of the sputum are more likely to produce sputum sample adequate for inflammatory cell analysis. If the first sputum sample is adequate, subsequent samples are very likely to be adequate as well. If the first sputum sample is inadequate, the quality of subsequent samples cannot be predicted, since there are similar probabilities of having adequate or inadequate samples. Monaldi Arch Chest Dis 2007; 67: 2, 81-83.

Keywords: Induced sputum, sputum quality, asthma, COPD.

Cardiothoracic Department, Respiratory Pathophysiology, University of Pisa, Italy.

Correspondence: Dr. Maria Laura Bartoli, Dipartimento Cardiotoracico, Fisiopatologia Respiratoria, Ospedale di Cisanello, Via Paradisa 2, 56124 Pisa, Italy; e-mail: m.bartoli@ao-pisa.toscana.it

\section{Introduction}

In the last few years analysis of induced sputum has been increasingly adopted in the evaluation of airway inflammation in respiratory diseases [1]. Induced sputum is a safe and well tolerated method [2] of collecting inflammatory cells and mediators from the airway lumen and can be easily repeated several times by the same subject. The adequacy of sputum slides is important in ensuring the reliability of the method. As about $20 \%$ of sputum samples do not allow an adequate differential cell count to be performed $[3,4]$, it would be useful to find predicting factors for sputum quality. We have stated that sputum quality is only in part affected by clinical and functional characteristics of subjects [4]. However, we now wonder if the presence of sputum productive cough during the days preceding the test, the easiness in sputum collection, the aspect of the sample collected, or the quality of the first sputum in subjects who repeat- ed the test several times, can be used as predicting factors of sputum adequacy.

\section{Methods}

We examined retrospectively 547 sputum samples obtained from 238 subjects with asthma or COPD of different degrees (mean age: $42 \pm 13 \mathrm{yrs}$, $39 \%$ smokers, $\mathrm{FEV}_{1}: 90 \pm 26 \%$ predicted). Sputum samples were collected either spontaneously $(n=184)$ or after induction with hypertonic saline inhalation $(n=363)$. In a group of 140 patients who repeated sputum collection one or more times, we checked out if patients with inadequate first sputum produced more inadequate samples in the following tests than patients whose first sputum was adequate.

Sputum induction and processing were performed as previously described [5]. Sputum was induced by hypertonic saline (3-5\%) nebulisation $(2.8$ $\mathrm{ml} / \mathrm{min}$ output), after inhaled $\beta 2$-agonist pretreat- 
ment. At the time of sputum induction, patients were asked about the presence of a productive cough during the days preceding the test, and the easiness in sputum collection during the test. A short questionnaire was administered by one of the two nurses attending the test (table 1). Before sending the sputum sample to the laboratory for processing, sputum samples were recorded by the same nurse as having good or bad macroscopic aspect according to the presence of visible plugs and viscid portions.

In the laboratory, sputum samples were processed by experienced technicians, using the whole sample method [4]. Only slides with viability greater than $50 \%$, at least 300 inflammatory cells counted, with good or fair integrity of cells and cell distribution, were considered as adequate. Interobserver reproducibility for differential cell counts (25) and for quality evaluation (35) were assessed on slides randomly selected, evaluated by Intraclass Correlation Coefficient (RI) and Cohen concordance test respectively and was reported as good [4].

\section{Results}

Out of 547 sputum samples, 409 were adequate $(75 \%)$ and 138 inadequate. We found no significant difference in adequacy percentages among induced and spontaneous sputum samples $(77.0 \%$

Table 1. - Short questionnaire completed by the patient and the nurse at the time of sputum induction

For the patient:

- Have you had cough and sputum on most days in the last two weeks?

$$
\text { Yes }
$$$$
\text { No }
$$

- The collection of sputum during and after saline inhalation was:

Easy

Fairly difficult

Definitely difficult

For the nurse:

- The macroscopic aspect of the sputum sample was:

Good (presence of plugs)

Bad (absence of any visible plug) vs $71.3 \%$ ). A higher percentage of adequate samples was observed when subjects reported productive cough in the days preceding the test (n:197), than when no phlegm was reported (n:350) $(82 \%$ vs $70 \%, p=0.003)$. This was true for spontaneous ( $82 \%$ vs $47 \%, p=0.0001$ ), but not for induced sputum samples $(83 \%$ vs $75 \%, \mathrm{p}=0.18)$. Patients reporting easiness in expectoration (n:279) produced more adequate samples than those reporting difficulty in expectoration (n:268) (85\% vs 63\%, $p=0.0001)$, irrespective of the type of samples (spontaneous or induced). $91 \%$ of sputum samples with a "good" macroscopic aspect gave adequate slides, while only $38 \%$ of samples with a "bad" macroscopic aspect gave adequate slides ( $p=0.0001)$, irrespective of the type of samples (spontaneous or induced sputum). A positive and negative predictive values for the factors considered are reported in table 2 . Positive predictive value was high for all factors, particularly for macroscopic aspect of induced samples. A negative predictive value was in general low for all factors. Spontaneous samples showed however a higher negative predictive value for macroscopic aspect. The combination of all three different factors brought to a very high positive predictive value, particularly in induced sputum, and increased negative predictive value, particularly in spontaneous samples.

Considering the adequacy of the first sputum sample among a group of 140 patients who repeated the test one or more times, we found that subjects with an inadequate first sample $(n=40)$ had a lower percentage of adequate samples in the subsequent tests (41 of 91 samples, $45 \%$ ) than patients $(n=115)$ with adequate first sample (172 of 187 samples, 92\%) $(p=0.0001)$.

\section{Discussion}

The novelty of the present study is to attempt to find some simple clinical factors which can predict the quality of the sputum samples obtained in a large group of asthmatic subjects. To our knowledge, this aspect has not been investigated in any methodologic publication on this topic. This information might be useful in sparing time in processing samples with very low predictive quality value.

Table 2. - Predictive values of the parameters considered

\begin{tabular}{lcccccc}
\hline & All sputum samples & \multicolumn{2}{c}{ Induced samples } & \multicolumn{2}{c}{ Spontaneous samples } \\
\hline & PPV & NPV & PPV & NPV & PPV & NPV \\
\cline { 2 - 7 } & $81.8 \%$ & $30.0 \%$ & $83.3 \%$ & $25.0 \%$ & $82.7 \%$ & $52.6 \%$ \\
Presence of productive cough & $85.1 \%$ & $35.9 \%$ & $91.5 \%$ & $44.2 \%$ & $80.5 \%$ & $55.6 \%$ \\
Easiness in expectoration & $90.5 \%$ & $60.0 \%$ & $94.8 \%$ & $58.6 \%$ & $83.2 \%$ & $66.7 \%$ \\
Good macroscopical aspect of the sample & & & & & & \\
Productive cough + easiness in expectoration & $91.4 \%$ & $66.9 \%$ & $97.9 \%$ & $62.6 \%$ & $88.6 \%$ & $84.2 \%$ \\
\hline
\end{tabular}

PPV: positive predictive value; NPV: negative predictive value. 
The presence of a productive cough in the days preceding the test and the easiness in expectoration makes it easier to collect material from the airway lumen and the assay of a good quality of sputum cell slides. This fact is particularly evident for spontaneous sputum. A previous paper has shown that spontaneous and induced sputum, collected in the same patients, had a similar cell composition, but that spontaneous sputum was associated with lower success rate and lower cell viability [6]. Our results are in agreement with this previous observation, as spontaneous sputum leads to a high quality rate only in subjects with productive cough in the day preceding sputum collection. In the opposite situation, spontaneous sputum should not be tried, because this can lead to a very low rate of adequate sputum samples.

The highest positive predictive value for a good quality of slides was obtained by the macroscopic aspect of sputum before processing, which is obviously related to the presence of phlegm in the days before and to the easiness in expectoration. Thus, the visual observation of plugs in the collected sputum is highly predictive of a good quality of sputum slides. Otherwise, the absence of sputum plugs has a very low negative predictive value, so the processing of the sample in these cases can not be avoided, since the probability of obtaining a good quality sample is however consistent. Only when spontaneous sputum and all predictive factors are taken toghether, the negative predictive value is high enough to exclude samples from processing. Patients whose first sputum is adequate, produce adequate sputum samples in a high percentage of the following tests (92\%), while patients whose first sputum is inadequate produce only $45 \%$ of adequate subsequent sputum samples. This fact underlines that some individual characteristics (e.g. easiness to expectorate) can explain the different success rate of sputum collection.

We used the whole sample method because this is one of the two well accepted techniques recommended for the sputum processing, with similar feasibility, accuracy and repeatability [7]. Furthermore, the larger volume of supernatant obtained by the whole method in comparison with the plug method can allow the measurements of a large number of soluble mediators in the sputum supernatant. The use of plug methods, which is generally associated with a higher quality of the slides, might have determined a lower rate of inadequate samples, thus reducing the possibility of finding significant correlations with the predictive factors considered.

All these considerations can help to predict whether a sputum sample will give adequate slides after processing.

\section{References}

1. Wark PA, Gibson PG. Clinical usefulness of inflammatory markers in asthma. Am J Respir Med 2003; 2: 11-19.

2. Wong HH, Fahy JV. Safety of one method of sputum induction in asthmatic subjects. Am J Respir Crit Care Med 1997; 155: 299-303.

3. Jones PD, Hankin R, Simpson J, Gibson PG, Henry RL. The tolerability, safety, and success of sputum induction and combined hypertonic saline challenge in children. Am J Respir Crit Care Med 2001; 164: 11461149.

4. Bartoli ML, Bacci E, Carnevali S, et al. Quality evaluation of samples obtained by spontaneous or induced sputum: comparison between two methods of processing and relationship with clinical findings. J of Asthma 2002; 36, 6: 479-486.

5. Bacci E, Cianchetti S, Paggiaro PL, et al. Comparison between hypertonic and isotonic saline-induced sputum in the evaluation of airway inflammation in subjects with moderate asthma. Clin Exper Allergy 1996; 26: 13951400.

6. Pizzichini MM, Popov TA, Efthimiadis A, et al. Spontaneous and induced sputum to measure indices of airway inflammation in asthma. Am J Respir Crit Care Med 1996; 154: 866-9.

7. Spanevello A, Beghe B, Bianchi A, Migliori GB, et al. Comparison of two methods of processing induced sputum: selected versus entire sputum. Am J Respir Crit Care Med 1998; 157: 665-8.

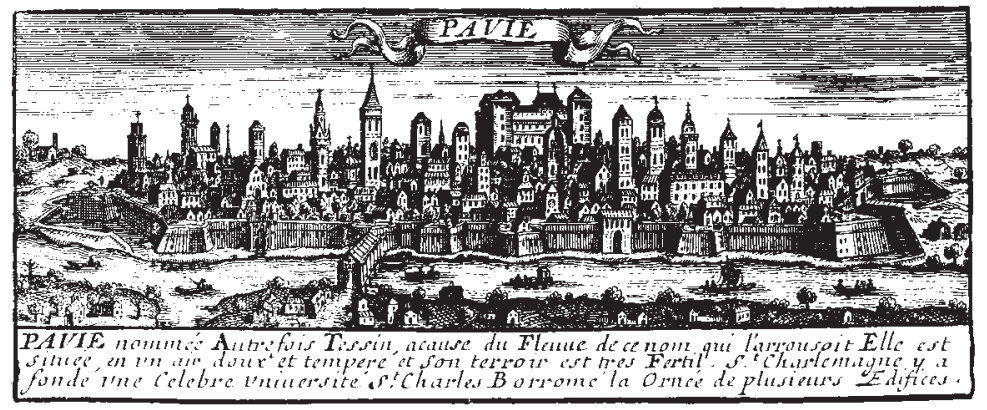

